\title{
Los mercados locales alternativos en México y Colombia: resistencias y transformaciones en torno a procesos
}

de certificación*

Cómo citar este artículo: Roldán Rueda, H. M., Gracia, M. A., y Mier y Terán, M. (2018). Los mercados locales alternativos en México y Colombia: resistencias y transformaciones en torno a procesos de certificación. Cuadernos de Desarrollo Rural, 15 (82), I-I7. https://www.doi.org/I0.III44/Javeriana.cdri5-82.mlam

Héctor Nicolás Roldán Rueda

El Colegio de la Frontera Sur, México

ORCID: http://orcid.org/0000-0003-3726-5910

María Amalia Gracia

El Colegio de la Frontera Sur, México

magracia@ecosur.mx

ORCID: http://orcid.org/0000-0002-1920-5618

Mateo Mier y Terán

El Colegio de la Frontera Sur, México

ORCID: http://orcid.org/0000-000I-6512-7238

DOI: https://doi.org/I0.III44/Javeriana.cdrı5-82.mlam

Redalyc: http://www.redalyc.org/articulo.oa?id=II757724004

Recibido: 13 marzo 2018 I Publicación: I8 Diciembre 2018

\section{Resumen:}

Una de las principales dificultades que tienen pequeños y medianos productores en Colombia y México es el acceso a mercados favorables. Atendiendo a esta problemática, distintos actores han generado estrategias de comercialización para recuperar espacios físicos y simbólicos que expresan demandas y reivindicaciones asociadas a la producción, la distribución y el consumo de alimentos locales. A partir de técnicas de investigación cualitativa, estudiamos mecanismos utilizados por cuatro mercados locales alternativos de estos dos países para diferenciar su producción, así como los sujetos involucrados; los resultados muestran las virtudes, las tensiones y los retos que suscitan los procesos de certificación.

Palabras clave: certificación, mercados locales, confianza, sistema agroalimentario. 


\title{
Alternative Local Markets in Mexico and Colombia: Resistance and Transformations Regarding the Certification Processes
}

\begin{abstract}
:
One of the main difficulties for medium- and small-sized food producers both in Colombia and Mexico is the access to favorable markets. Due to this problem, different actors have produced marketing strategies intended to recover the physical and symbolic spaces expressing both the demands and claims related to the production, distribution and consumption of local food. Based on qualitative research techniques, we studied the mechanisms used by four alternative local markets in these two countries, in order to differentiate their production as well as the subjects involved. The results show the benefits, tensions and challenges stemming from the certification processes.
\end{abstract}

Keywords: certification, local markets, trustworthiness, agricultural-food system.

\section{Introducción}

Los mercados locales alternativos (Goodman, Goodman y Dupuis, 20I2) surgidos en las últimas dos décadas en ciudades latinoamericanas se caracterizan por la posibilidad de consolidar espacios de intercambio favorables para productores, transformadores, intermediarios y consumidores. Se les denomina locales por los vínculos sociales, políticos y económicos que crean en los territorios en los que se ubican y por la cercanía que tienen con quienes los abastecen, pues, de acuerdo con lo expresado por miembros de estos mercados, la mayoría de los productores que participan recorren entre 20 y 250 kilómetros; la nominación alternativos refiere al tipo de relaciones, objetivos y transformaciones que plantean estos espacios atendiendo a los contextos en los que se ubican (Barriga, 2016; Kay, 2016; Maela, 2012).

Estos mercados cuestionan las lógicas del sistema agroalimentario convencional globalizado y recuperan espacios locales (Delgado, 20ro). Dicha recuperación reconecta la producción y el consumo -incluso reconstruyendo el papel de la intermediación-, redefine los valores que motivan la participación y redistribuye excedentes, con lo que abona al fortalecimiento de economías locales (Rosset, 2007).

En estos espacios se expresan demandas, reivindicaciones y disputas más amplias que convergen en discursos y formas de incidencia política a nivel local en torno al campesinado y al sistema agroalimentario hegemónico -y su propuesta de alimentación-; dichas expresiones retoman nociones de justicia, solidaridad, confianza, respeto y reciprocidad, las cuales se definen a partir de su integración en las relaciones sociales y estimulan el reconocimiento de la otra persona como parte esencial de la transformación de la política, la economía y la sociedad (Coraggio, 2004).

A partir de información recabada en cuatro mercados locales de México y Colombia, nos enfocamos en aquellos procesos de certificación ( $\mathrm{PdC}$ ) cuyos mecanismos inciden en las prácticas productivas -al promover la eliminación de insumos tóxicos para la salud y el medio ambiente- y transforman las prácticas organizativas, de distribución y de consumo, con el objetivo de analizar los mecanismos diseñados para su implementación, así como los efectos y las posturas que generan en las prácticas de producción, distribución y consumo que dinamizan sus participantes. 
Mientras los $\mathrm{PdC}$ promovidos por agencias privilegian canales de comercialización convencionales incluyendo la exportación de productos orgánicos-, los $\mathrm{PdC}$ implementados en los mercados analizados promueven los intercambios directos (Sevilla y Soler, 20I2), la transición hacia prácticas agroecológicas (Calle, Vara y Cuéllar, 2013), el intercambio de saberes y la incorporación de valores y principios de confianza, respeto y solidaridad en las etapas del proceso productivo - apropiación, producción, distribución y consumo (Toledo 1992)-.

Aun dentro de los mercados locales alternativos, entendemos que los PdC generan posturas diversas a raíz de sus antecedentes, los actores que los promueven, las consecuencias y los beneficios que traen y, sobre todo, por la lógica a partir de la cual se implementan (Carlisle, 2015). Si bien este tipo de procesos permite consolidar espacios y redes diferenciadas para la producción y comercialización de algunos productos -entre los que sobresalen variedades de hortalizas, frutas, café y miel-, en ocasiones esta diferenciación no alcanza a favorecer a los pequeños y medianos productores - actores clave en los discursos que motivan estas experiencias-. Lo anterior puede alejar a los mercados locales de su meta de acercar al productor con el consumidor y terminar generando "espacios de refugio para minorías” (Calle, Soler y Rivera, 20II), tanto del lado de la producción como del consumo, como se observa en distintos nichos de mercado.

De allí que en el análisis sea necesario identificar y diferenciar la producción y circulación de productos alternativos de la producción y circulación alternativa de productos; mientras la primera se basa en la satisfacción de necesidades sin cuestionar las lógicas que las definen, la segunda apunta a transformaciones más amplias de carácter social, económico, político, cultural y ambiental.

Ubicamos las experiencias analizadas dentro de la segunda categoría, en la medida que su emergencia y consolidación parte de una fuerte crítica a los sistemas agroalimentarios convencionales y de la necesidad de incorporar principios de justicia, solidaridad y respeto dentro de cada una de las etapas del proceso productivo. En ese sentido, los mecanismos de certificación diseñados por este tipo de mercados se han convertido en una oportunidad para reconstruir el vínculo entre la producción y el consumo, así como para reconfigurar las relaciones sociales basadas en la confianza, por lo que se generan escenarios favorables - aun sin estar libres de tensiones y contradicciones- para pequeños y medianos productores en términos sociales, políticos y económicos.

Este artículo se divide en dos partes: en la primera, se presenta la perspectiva analítica y metodológica y se contextualizan y caracterizan los mercados analizados; en la segunda, se discuten los resultados más relevantes, enfocados en la forma en que se abordan los $\mathrm{PdC}$, a fin de contrastar las posturas que estos mecanismos inauguran y refuerzan a partir de la experiencia de los sujetos que participaron de la investigación.

\section{Perspectiva analítica}

La consolidación de espacios alternativos para la comercialización, la distribución y el consumo de alimentos constituye un referente para la transformación de los sistemas agroalimentarios en la medida en que dichos espacios expresan demandas y visibilizan las luchas y reivindicaciones de sus participantes (Crespo y Sabín, 20I4; Kay, 2016). Un sistema agroalimentario se define como "el conjunto de las actividades que concurren a la formación y a la distribución de los productos alimentarios y, en consecuencia, al cumplimiento de la función de la alimentación humana en una sociedad determinada" (Malassis, 1973, p. I2). Su estudio permite entender cómo se abastece de alimentos una sociedad, las relaciones que se establecen entre los actores que intervienen en dicho proceso y las consecuencias sociales, políticas y económicas que se derivan de lo anterior (Tarditti, 20I2).

Un elemento diferenciador de la forma en que se consolidan las relaciones dentro de los mercados alternativos locales tiene que ver con las lógicas y los cuestionamientos que plantean sus participantes frente al mercado capitalista, pues la "principal diferencia entre los mercados locales y el sistema de mercado es que los primeros 
fomentan las relaciones sociales, mientras que el mercado capitalista disuelve los lazos de dependencia entre los miembros de una comunidad y fomenta el individualismo” (Santana, 2008, p. I29). Además de buscar la generación de excedentes para su sostenimiento, su objetivo es incorporar acciones solidarias, recíprocas y de redistribución mediadas por diversos actores y vínculos sociales.

Al mismo tiempo, su emergencia permite el fortalecimiento de redes de intercambio comercial, de saberes y de información en diferentes niveles - local, regional, nacional o mundial- que posibilitan estrategias y fortalecen procesos complementarios para sus participantes, como escuelas campesinas, finanzas solidarias y relevo y complementariedad generacional y de género, entre otros (Caballero, Dumraf, Gonzales, Mainella y Moricz, 2010). El estudio simultáneo de estas formas de organización social, junto con las estrategias de producción, distribución y consumo, permite visibilizar otras estructuras sociales de intercambio no regidas exclusivamente por los principios de la economía neoliberal (Gil, 20I4), lo que en términos políticos

supone demostrar que las relaciones sociales establecidas por la economía de mercado son tan solo una opción histórica para la construcción del orden y la sociabilidad humana, y que por tanto han existido y podrían existir formas alternativas de organización social. (Lahera, 1999, p. 32)

Por esta razón, explorar las diferencias entre los mecanismos que integran los $\mathrm{PdC}$ en diversos espacios de comercialización e intercambio alternativos requiere conocer el tipo de prácticas productivas y organizativas, además de las relaciones de intercambio que se construyen y el tipo de mercados que se generan (Soler y Calle, 20I0). También es necesario visibilizar los procesos complementarios-organizativos, de financiación, producción, transformación, distribución y consumo que se llevan a cabo y que dan lugar a transformaciones estructurales que habilitan alternativas sociales, políticas, económicas, ambientales o culturales.

Sin embargo, las alternativas planteadas pueden ser integradas dentro de la producción convencional, como se observa en la inclusión de productos con certificados de producción orgánica -o de mercado justo- dentro de canales convencionales. Lo anterior se traduce en un aumento de la oferta de productos certificados que, lejos de desafiar o cuestionar las estructuras convencionales a nivel productivo, organizativo o de comercialización (Tarditti, 20I2), terminan siendo cooptados y articulados por discursos hegemónicos que desintegran la politicidad de este tipo de experiencias (Gracia, 2015).

Al respecto, la emergencia de espacios locales de comercialización sin vínculos territoriales puede ser interpretada como procesos de convencionalización "en los que los proyectos no sean sino la ampliación y recreación de nichos de mercado hacia consumidores 'alternativos'” (Calle et al., 20II, p. 224) que basan sus estrategias en la diferenciación social y de salud y el culto al cuerpo. Se refuerzan así mecanismos convencionales de producción y comercialización de productos alternativos, que se abastecen de procesos de agricultura de sustitución de insumos, uso de tecnologías y concentración de tierras y recursos (Rosset, 2007).

Ante este fenómeno, una virtud de este tipo de mercados es la recuperación del sentido político de lo local, en la medida en que este carácter aporta elementos para la consolidación de procesos que no se agotan en la adjetivación de los productos y los espacios de comercialización, sino que plantean alternativas y estrategias de producción, circulación y consumo que permiten identificar las formas en las que se confrontan los "valores hegemónicos de la ética del mercado, poniendo en práctica valores alternativos” (Caballero et al., 2010, p. 33), en un contexto en el que la confianza, la reciprocidad y la solidaridad son valores presentes en las relaciones sociales que se dan en estos espacios.

En ese sentido, analizar estos espacios de manera situada, es decir, "considerando que los mismos ocurren en contextos históricos y espaciales específicos y están protagonizados por una constelación plural y diversa de grupos, sujetos, organizaciones y movimientos [...] que resisten colectivamente de manera más o menos consciente, más o menos defensiva, los efectos devastadores de las distintas crisis” (Gracia, 2015, p. I8), permite identificar la singularidad de cada experiencia frente a los procesos sociales, políticos y económicos que enfrentan. 
Lo anterior supone que un mismo fenómeno puede ser muy diferente según la historicidad de los sujetos que lo dinamizan (Zemelman, 2006), lo que da lugar a modelos de organización social caracterizados por interacciones, negociaciones y forcejeos sociales que tienen lugar entre varios tipos de actores, no solo de los presentes en ciertos encuentros cara a cara, sino también de los ausentes que influyen en la situación y afectan las acciones y los resultados (Long, 2007).

Considerando sus particularidades, la consolidación de cada experiencia puede ser interpretada como una conquista espacial y simbólica en la que las formas de poder hegemónicas son confrontadas en diferentes contextos y dan lugar a demandas que se convierten en discursos políticos y formas de incidencia más contundentes, como puede ser su participación en la formulación de políticas públicas, leyes, reconocimiento político, entre otras (Lamine, Darolt y Brandenburg, 20I2). Ejemplo de ello son algunas de las estrategias que han implementado las experiencias analizadas a partir de su participación activa en la elaboración de políticas públicas, marcos legales y visibilización del campesinado en México y en Colombia (Parrado y Molina, 20I4; Roldán-Rueda y Gracia, 2015).

Los factores que inciden en la configuración de estos mercados son diversos y responden a las realidades micro y macrosociales, que, sin necesidad de ser evidentes para sus participantes, determinan sus estrategias y la construcción de nuevas identidades sociales y políticas (Long, 2007). Los mercados locales se constituyen -o poseen la potencialidad de constituirse - en un mecanismo de reforzamiento o generación de la identidad campesina, no solo por los discursos reivindicativos que apelan a la soberanía y autonomía alimentaria, la justicia social y ambiental o los alimentos libres de tóxicos, sino también porque, al generar procesos, posturas y sentidos distintos, expresan las raíces campesinas de sus protagonistas (León, Valdez y Vásquez, 200z).

A partir de las luchas y reivindicaciones planteadas desde los mercados, las prácticas que dinamizan sus participantes comienzan a fortalecer diversas formas de hacer y pensar la economía, de consolidar economías que vayan más allá de lo estrictamente económico al dotar de otro sentido a las acciones cotidianas individuales (Coraggio, 2004), lo que otorga sentidos identitarios y significados diversos a los espacios físicos y simbólicos que giran en torno a la alimentación como ejercicio colectivo. Por lo tanto, no se trata solamente de tomar conciencia del espacio, sino de tomar conciencia de la posibilidad de construir sentido desde ese espacio (Zemelman, 2006).

Adicionalmente, el tipo de vínculos y formas de participación en redes y procesos complementarios afianza relaciones sociales en las que los participantes tienen la capacidad de decidir y de autoorganizarse y contribuye con procesos que se estimulan de abajo hacia arriba a partir de intereses y necesidades locales que logran trascender a escalas más amplias (Beckie, Kennedy y Wittman, 2012). Dicho escalamiento se logra cuando "las acciones de múltiples agentes en interacción dinámica y siguiendo reglas locales en lugar de órdenes de arriba-abajo generan algún tipo de macrocomportamiento o estructura visible” (Escobar, 2010, p. 302), lo cual permite que los procesos se transformen y amplíen respetando sus particularidades.

Las relaciones, las articulaciones y los vínculos que identificamos en los mercados se ponen en juego en los PdC, que sirven para ejemplificar la incidencia de los factores externos en la construcción de un territorio, el sentido que le otorgan sus participantes y las escalas más amplias que involucran, ya sea de manera activa o como formas de adaptación frente a los procesos y las tendencias que los interpelan. 


\section{Metodología y mercados seleccionados}

\section{Aspectos metodológicos}

Al tratarse del estudio de un fenómeno contemporáneo, así como por el tipo de indicadores y unidades de análisis que se definieron (tabla I), el diseño seleccionado fue el estudio de caso, que requiere la integración entre los datos empíricos, las fuentes de información secundaria (informes, censos, entrevistas...) y los postulados teóricos que se integran en las diferentes etapas de la investigación (Yin, 1994). Teniendo en cuenta que se trata de procesos que se consolidan en las mismas o similares circunstancias estructurales - pero que desarrollan formas sociales diferentes-, fue necesario incluir en el diseño y análisis estrategias que permitieran comprender la forma en que se "producen, reproducen, consolidan y transforman tales diferencias e identificar los procesos sociales involucrados, y no solo los resultados estructurales” (Long, 2007, p. 108).

Las unidades de análisis que delimitan cada uno de los casos se definieron a partir de la participación de los sujetos dentro de los mercados locales seleccionados, así como de los vínculos o procesos complementarios que generan con redes y procesos externos, que dan cuenta de articulaciones más amplias. Como categorías de análisis, definimos las prácticas organizativas, productivas y de comercialización e intercambio, además de los apoyos y las alianzas que articulan los mercados seleccionados. Estas categorías están integradas por indicadores que exploran algunas de las relaciones, estrategias, sentidos y dinámicas en diferentes niveles, tanto a nivel de mercado, en las relaciones con actores externos, como individual, en las prácticas y decisiones que se toman dentro de la unidad de producción.

TABLA 1

Caso: mercados locales en México y Colombia

\begin{tabular}{lll}
\hline \multicolumn{1}{c}{ Unidades de análisis } & \multicolumn{1}{c}{$\begin{array}{c}\text { Categorías de } \\
\text { análisis }\end{array}$} & \multicolumn{1}{c}{ Indicadores } \\
\hline $\begin{array}{l}\text { Participación de } \\
\text { sujetos sociales en } \\
\text { mercados locales }\end{array}$ & $\begin{array}{l}\text { Prácticas } \\
\text { organizativas }\end{array}$ & $\begin{array}{l}\text { - Tipo de organización } \\
\text { - Fortalecimiento/estructura organizativa } \\
\text { - Formas de participación }\end{array}$ \\
\cline { 2 - 3 } $\begin{array}{l}\text { Redes, vínculos o } \\
\text { procesos } \\
\text { complementarios }\end{array}$ & productivas & - Tipo de producción/productor \\
& & $\begin{array}{c}\text { - Diversificación y transformación de } \\
\text { productos }\end{array}$ \\
& & - Vínculos con la comercialización \\
& Prácticas de & - Procesos en los que participa \\
\hline comercialización e & - Tipo de intermediación \\
& - Espacios de comercialización \\
& complementarios \\
\cline { 2 - 3 } & Apoyos y alianzas & - Mecanismos de fijación de precios \\
\hline & & - Formas de apoyo y participación \\
& & - Tipo de proyectos y programas \\
& & Tipos y duración de los apoyos \\
\hline
\end{tabular}

Fuente: elaboración propia 
Durante las etapas de la investigación, se privilegió el uso de herramientas y estrategias cualitativas, y, en menor medida, de información cuantitativa, para comprender los contextos más amplios en los que se ubican los mercados y las redes en las que participan (tabla 2). Para la recolección de la información, se diseñaron entrevistas semiestructuradas, diálogos informales y guías de observación; para la sistematización y el análisis de la información, se utilizó el programa de análisis cualitativo Atlas.ti. 7.5, el cual permitió contrastar información e integrar categorías emergentes a partir de las relaciones existentes entre códigos no contempladas en el diseño metodológico.

TABLA 2

\begin{tabular}{|c|c|c|c|}
\hline País & Mercado local & Ubicación & Red o vínculo \\
\hline \multirow[t]{3}{*}{ México } & Mercado de Productos Naturales & San Luis & \multirow{3}{*}{$\begin{array}{l}\text { Red Mexicana de } \\
\text { Tianguis y Mercados } \\
\text { Orgánicos }\end{array}$} \\
\hline & Macuilli Teotzin & Potosí & \\
\hline & Tianguis Alternativo de Puebla & Puebla & \\
\hline \multirow[t]{2}{*}{ Colombia } & Mercados Campesinos & Bogotá & Mercados Campesinos \\
\hline & Agrosolidaria & $\begin{array}{l}\text { Bogotá } \\
\text { (Engativá) }\end{array}$ & Agrosolidaria \\
\hline
\end{tabular}

Fuente: elaboración propia

En cada una de las etapas, se utilizaron fuentes de información secundarias, entre las que sobresalen los censos y las encuestas agrícolas presentados por instituciones oficiales de cada país, los marcos legales que abordan temas relacionados con los $\mathrm{PdC}$, así como investigaciones académicas, informes y reportes de diversas organizaciones (Cuéllar, 20ı0; Giraldo y Rosset, 20ı6; Zamilpa, 2014).

La información se recabó entre abril y agosto de 20I6, en Colombia, y entre marzo y julio de 2017, en México, luego de haber realizado salidas exploratorias en las que se identificaron las experiencias que integran esta investigación. Estas salidas permitieron identificar los roles de quienes participan de estos mercados: productores, consumidores, intermediarios, promotores, organizaciones de apoyo-actores externos, a partir de los cuales se diseñaron las herramientas metodológicas.

Este diseño metodológico permitió evidenciar elementos comunes expresados de maneras diversas por los participantes, teniendo en cuenta que los mercados analizados son singulares no solo por la especificidad de su contexto, sino también por el tipo de actores que se involucran en los diferentes procesos que vienen dinamizando.

\section{Contexto de los mercados seleccionados}

Los mercados analizados en este trabajo se caracterizan por plantear alternativas a problemáticas que enfrentan pequeños y medianos productores. Dentro de sus planteamientos, uno de los temas centrales es la incorporación de mecanismos para certificar los procesos de producción, de manera tal que garanticen la calidad de los productos y permitan su diferenciación frente al mercado. Las distintas posturas que estos procesos han ido generando en los participantes varían de acuerdo con sus trayectorias, contextos y el tipo de personas involucradas en cada uno de ellos.

Los mercados analizados en México, el Mercado de Productos Naturales Macuilli Teotzin, ubicado en la ciudad de San Luis Potosí (centro del país), y el Tianguis Alternativo de Puebla (la palabra tianguis viene del náhuatl tianquiztli, que quiere decir mercado), ubicado en la capital del Estado de Puebla (centro oriente), contaron entre 2004 y 2013 con la participación de la Red Mexicana de Tianguis y Mercados Orgánicos, desde la que se 
realizaron los primeros esfuerzos por consolidar espacios de comercialización de productos orgánicos abastecidos por pequeños y medianos productores. Al mismo tiempo, fue desde esta red que se comenzaron a implementar PdC participativa en cada uno de los mercados que la integraban.

Los avances en estos procesos fueron retomados por instancias oficiales, con el ánimo de integrar este tipo de certificaciones dentro del marco legal -ley de producción orgánica de 2006 y lineamientos a la ley de producción orgánica de 20I3-, lo que puede interpretarse en el contexto del crecimiento de la agricultura orgánica en el país y de la participación de pequeños y medianos productores (Gómez, Schwentesius, Ortigoza y Gómez, 20ro). Sin embargo, a partir de la entrada en vigor de dicho marco normativo en 2013 , la mayoría de los más de 35 mercados que integraban la red adoptaron posturas diversas que dieron lugar a su distanciamiento respecto a la normativa y a los PdC promovidos por la red, los cuales se traducían en la pérdida de autonomía y de capacidad para diseñar mecanismos de acuerdo con las particularidades de cada mercado (Roldán-Rueda, Gracia, Santana y Horbath, 2016).

A partir del trabajo que realizó la red y del interés de algunos productores y promotores por afianzar los PdC de acuerdo con lo estipulado en el marco legal, el Macuilli Teotzin se convirtió en el primer mercado en todo el país con la facultad de otorgar certificados de producción orgánica a través de PdC participativa, reconocimiento que lograron en marzo de 2018.

Por otro lado, el Alternativo de Puebla ha diseñado uno de los PdC más reconocidos a nivel nacional sin que dentro de sus prioridades esté la validación por parte de las instancias oficiales; sin embargo, la posibilidad de validar su PdC ante las instituciones oficiales es un tema que se discute entre sus participantes. Hace más de diez años, el Alternativo de Puebla viene consolidando un comité de certificación integrado por los participantes del mercado, quienes, con el paso del tiempo y de manera autónoma, han perfeccionado sus propios indicadores, lineamientos y recomendaciones para la producción. Al mismo tiempo, han diseñado estrategias para acercarse a los consumidores por medio de talleres, charlas y espacios de diálogo.

Por otro lado, el primer caso que abordamos en Colombia, denominado por sus participantes como Mercados Campesinos, ha sido una de las experiencias más exitosas de organización campesina y está conformada por nueve organizaciones campesinas de la región central del país -Cundinamarca, Boyacá, Tolima y Meta (Parrado y Molina, 20I4)-. El proceso comenzó a gestarse en 2003, y dentro de sus logros están la visibilización de la importancia de la economía campesina para la capital del país y el establecimiento de mercados, con cierta regularidad, en ocho parques de la ciudad.

Este proceso se transformó en 20ı6, a raíz del cambio de autoridades locales en la capital y de la posterior terminación de los acuerdos y permisos. Pese a ello, las organizaciones campesinas continúan fortaleciendo procesos organizativos, productivos y de comercialización en las regiones en donde se ubican los productores. Al mismo tiempo, diseñaron estrategias complementarias que han permitido en algunos casos dar continuidad al trabajo que venían desarrollando (mercados locales en zonas rurales, escuelas campesinas o encuentros regionales entre productores).

El segundo caso, la Confederación Agrosolidaria, es un modelo de organización socioeconómica que desde 1994 integra familias agricultoras y prosumidoras -término que incluye simultáneamente prácticas de consumo y producción- urbanas y rurales y que se viene fortaleciendo a partir de procesos organizativos locales en diferentes regiones. Con sede en el departamento de Boyacá (centro-oriente del país), para 2014 Agrosolidaria tenía presencia en más de i8 departamentos del país y más de 32.000 familias asociadas. Esta iniciativa integra, dentro de sus intereses y objetivos, diversos procesos complementarios, entre los que sobresalen las finanzas solidarias y los grupos asociativos de producción, distribución, transformación y consumo de alimentos.

La propuesta metodológica y de organización de Agrosolidaria se basa en procesos colectivos caracterizados por la autonomía, la descentralización, la interdependencia, la equidad y la complementariedad, el relevo generacional y la gestión asociada. Estos pilares se configuran a partir de una estructura organizacional horizontal que 
comienza con las unidades socioeconómicas familiares conformadas por grupos asociativos en torno a financiación, producción, transformación, distribución y consumo, los cuales a su vez conforman las seccionales, que se agrupan a nivel regional en federaciones, y a nivel nacional, en una confederación.

En síntesis, a diferencia de México, en Colombia no identificamos un actor que articule experiencias en torno a los PdC a nivel nacional. Sin embargo, las redes a las que se integran los mercados seleccionados en los dos países han priorizado la sustitución de insumos tóxicos, la transición agroecológica, el intercambio de saberes y la búsqueda de canales de comercialización alternativos para mejorar las condiciones de acceso y el tipo de vínculos con los productores.

En la tabla 3 incluimos distintos elementos que caracterizan a estos mercados, entre los que destacamos el número y el tipo de participantes; un elemento común que tienen estos espacios de comercialización es la presencia mayoritaria de productores que ofrecen productos transformados (salsas, conservas, panes, jabones, entre otros) y alimentos preparados, en contraste con los productores agrícolas que venden, principalmente, variedades de hortalizas y frutas de temporada. Otro aspecto importante tiene que ver con los canales de comercialización complementarios que pueden ser generados por el mismo mercado o de manera individual y que se consolidan a raíz de la frecuencia de los encuentros y la necesidad de vender la producción, por lo que se recurre en ocasiones a los canales convencionales o incluso a intermediarios acaparadores.

TABLA 3

\begin{tabular}{|c|c|c|c|c|c|c|}
\hline Mercado & $\begin{array}{c}\text { Año de } \\
\text { surgimiento }\end{array}$ & $\begin{array}{l}\text { Origen de los } \\
\text { productores }\end{array}$ & $\begin{array}{l}\text { Frecuencia } \\
\text { de los } \\
\text { encuentros }\end{array}$ & $\begin{array}{l}\text { Canales de comercialización } \\
\text { complementarios }\end{array}$ & $\begin{array}{l}\text { Número de } \\
\text { participantes y } \\
\text { productos }\end{array}$ & Alianzas \\
\hline $\begin{array}{l}\text { Mercado de } \\
\text { Productos } \\
\text { Naturales } \\
\text { Macuilli } \\
\text { Teotzin }\end{array}$ & 2011 & $\begin{array}{l}\text { Cuatro zonas } \\
\text { del estado: el } \\
\text { Altiplano, la } \\
\text { Huasteca, la } \\
\text { Zona Media y } \\
\text { la Zona Centro }\end{array}$ & $\begin{array}{l}\text { Una vez al } \\
\text { mes }\end{array}$ & $\begin{array}{l}\text { Tienda Casa Garambullo, SLP; } \\
\text { mercado local Matehuala, SLP }\end{array}$ & $\begin{array}{l}28 \text { transformadores } \\
\text { (panes, salsas, } \\
\text { conservas, jabones, } \\
\text { licores, platillos } \\
\text { típicos); un } \\
\text { artesano, y } 4 \\
\text { productores } \\
\text { (hortalizas, frutas } \\
\text { de temporada, café, } \\
\text { miel, tortillas) }\end{array}$ & $\begin{array}{l}\text { - UASLP1 } \\
\text { - Sagarpa }{ }^{2} \\
\text { - Senasica } \\
\text { - Productores } \\
\text { - Promotores } \\
\text { - Consumidores }\end{array}$ \\
\hline $\begin{array}{l}\text { Tianguis } \\
\text { Alternativo } \\
\text { de Puebla }\end{array}$ & 2007 & $\begin{array}{l}\text { Puebla, } \\
\text { Cholula, } \\
\text { Guerrero, } \\
\text { Estado de } \\
\text { México, } \\
\text { Morelos, } \\
\text { Hidalgo }\end{array}$ & Sábados & Mercados locales cercanos & $\begin{array}{l}20 \text { transformadores } \\
\text { y otros (panes, } \\
\text { salsas, quesos, } \\
\text { jabones, platillos } \\
\text { típicos) y } 7 \\
\text { productores } \\
\text { (hortalizas, frutas } \\
\text { de temporada, café, } \\
\text { miel, huevos, } \\
\text { tortillas) }\end{array}$ & $\begin{array}{l}\text { - Productores } \\
\text { - Promotores } \\
\text { - Consumidores } \\
\text { - Sembrarte } \\
\text { A. C. }\end{array}$ \\
\hline $\begin{array}{l}\text { Mercados } \\
\text { Campesinos }\end{array}$ & $2007-2016$ & $\begin{array}{l}\text { Región central } \\
\text { de Colombia }\end{array}$ & $\begin{array}{l}\text { Cada } 15 \\
\text { dias (2007- } \\
2016)\end{array}$ & $\begin{array}{l}\text { Mercados locales en cada } \\
\text { región }\end{array}$ & $\begin{array}{l}15 \text { transformadores } \\
\text { y otros (quesos, } \\
\text { postres, embutidos, } \\
\text { platillos típicos) y } \\
12 \text { productores } \\
\text { (hortalizas, frutas, } \\
\text { papa, came, pollo) }\end{array}$ & $\begin{array}{l}\text { - Oxfam } \\
\text { - } \mathrm{CICC}^{4} \\
\text { - ILSA } \\
\text { - } \mathrm{SDE}^{6} \\
\text { - } \mathrm{UNAL}^{7}\end{array}$ \\
\hline $\begin{array}{l}\text { Agrosolidaria } \\
\text { (Engativá) }\end{array}$ & 2013 & $\begin{array}{l}\text { Productores de } \\
\text { la zona rural } \\
\text { de Bogotá y } \\
\text { las seccionales } \\
\text { de } \\
\text { Agrosolidaria }\end{array}$ & Intermitente & $\begin{array}{l}\text { Tienda virtual: } \\
\text { www.agrosolidariaengativa.org }\end{array}$ & $\begin{array}{l}15 \text { transformadores } \\
\text { y otros (embutidos, } \\
\text { snacks, alimentos } \\
\text { precocidos) y } 12 \\
\text { productores } \\
\text { (quinua, hortalizas, } \\
\text { frutas, papa, zetas) }\end{array}$ & $\begin{array}{l}\text { - Confederación } \\
\text { Agrosolidaria } \\
\text { - Fundación } \\
\text { Semillas } \\
\text { - } \text { RMABR }^{8}\end{array}$ \\
\hline
\end{tabular}

Fuente: elaboración propia

${ }^{\text {I } U n i v e r s i d a d ~ A u t o ́ n o m a ~ d e ~ S a n ~ L u i s ~ P o t o s i ́ ~}$

${ }^{2}$ Secretaría de Agricultura, Ganadería, Desarrollo Rural, Pesca y Alimentación

3 Servicio Nacional de Sanidad, Inocuidad y Calidad Agroalimentaria

4 Comité de Interlocución Campesina y Comuna

${ }^{5}$ Instituto Latinoamericano para una Sociedad y Derecho Alternativos

${ }^{6}$ Secretaria de Desarrollo Económico

7 Universidad Nacional de Colombia

${ }^{8}$ Red de Mercados Alternativos de Bogotá Región 


\section{Resultados}

Frente a una problemática en común, el acceso a mercados favorables, los mercados locales alternativos analizados han implementado distintas estrategias y mecanismos de participación en torno a los $\mathrm{PdC}$, lo cual incide en el tipo de relaciones que reproducen y, por tanto, en el tipo de mercado que recrean.

Uno de los objetivos principales de los PdC es garantizar la calidad de los productos, objetivo que se refuerza mediante las relaciones de confianza y de cercanía construidas entre productores y consumidores. Estos mercados expresan aspectos culturales, étnicos e identitarios de sus protagonistas, que se aprecian en sus formas de relacionarse y en el tipo de información, preocupaciones y alegrías compartidas entre los participantes, lo cual muestra que lo económico es tan solo una de sus virtudes. Para muchos de los protagonistas de estas experiencias, los vínculos que mantienen son los que dotan de sentidos a estos espacios: "Es necesario mantener y recuperar esa relación fraterna, esa armonía, entre el que compra y el que produce los alimentos, porque si se rompió esa relación, nos aislaron y todo se convierte en capital" (líder campesina de Mercados Campesinos, comunicación personal, julio de 20I6).

En ese sentido, los PdC que implementan estos mercados pueden ser interpretados como una estrategia que refleja los sentidos, las identidades y los motivos presentes en cada contexto, así como las formas en que se relacionan sus participantes, que permiten afianzar vínculos y proyectos en común. De allí se desprende que, además de garantizar la calidad de los productos, este tipo de PdC implementa mecanismos que buscan reconocer, antes que validar, el esfuerzo, la dedicación, el tiempo y la pasión con la que se produce (tabla 4), con lo cual se recupera el valor de la palabra y del diálogo a partir de la construcción de relaciones de confianza entre quienes participan de estos mercados. 
TABLA 4

MECANismos de CERTIFICACIÓN IMPLEMENTAdos POR LOS MERCADOS ANALIZAdos

\begin{tabular}{|c|c|c|c|}
\hline & \multicolumn{2}{|c|}{ México } & Colombia \\
\hline & $\begin{array}{l}\text { Macuilli } \\
\text { Teotzin }\end{array}$ & $\begin{array}{l}\text { Alternativo de } \\
\text { Puebla }\end{array}$ & $\begin{array}{l}\text { Agrosolidaria } \\
\text { (Engativá) }\end{array}$ \\
\hline $\begin{array}{l}\text { Certificación } \\
\text { participativa }\end{array}$ & $\begin{array}{l}\text { Tomando } \\
\text { como } \\
\text { referencia la } \\
\text { ley de } \\
\text { producción } \\
\text { orgánica de } \\
2006\end{array}$ & $\begin{array}{l}\text { Diseñada } \\
\text { colectivamente } \\
\text { a partir de las } \\
\text { caracteristicas } \\
\text { de los } \\
\text { participantes }\end{array}$ & $\begin{array}{l}\text { Existen esfuerzos incipientes } \\
\text { para la formalización de este } \\
\text { tipo de procesos; se concibe } \\
\text { como un elemento importante }\end{array}$ \\
\hline Sellos y etiquetas & $\begin{array}{l}\text { Etiquetas de } \\
\text { producción } \\
\text { orgánica } \\
\text { avaladas por } \\
\text { instancias } \\
\text { oficiales }\end{array}$ & \multicolumn{2}{|c|}{$\begin{array}{l}\text { Los productores diseñan etiquetas en las que } \\
\text { resaltan las características de sus productos } \\
\text { (origen, tipo de insumos y propiedades) }\end{array}$} \\
\hline Categorías & \multicolumn{3}{|c|}{$\begin{array}{l}\text { Remiten a la etapa en la que se ubica cada productor: } \\
\text { orgánico, agroecológico, en transición o convencional; } \\
\text { generalmente existe un distintivo en el espacio de cada } \\
\text { productor }\end{array}$} \\
\hline $\begin{array}{l}\text { Vinculados a } \\
\text { mercados similares }\end{array}$ & \multicolumn{3}{|c|}{$\begin{array}{l}\text { Es común que productores que han implementado PdC en } \\
\text { mercados similares puedan participar en estos y viceversa }\end{array}$} \\
\hline Confianza & \multicolumn{3}{|c|}{$\begin{array}{l}\text { Es un componente principal para realizar intercambios, ya que } \\
\text { no todos los consumidores se guían por la etiquetas }\end{array}$} \\
\hline $\begin{array}{l}\text { Prácticas } \\
\text { agroecológicas/escuelas } \\
\text { campesinas }\end{array}$ & \multicolumn{2}{|c|}{$\begin{array}{l}\text { Existen procesos } \\
\text { complementarios que } \\
\text { permiten el intercambio de } \\
\text { saberes, pero no es un eje } \\
\text { central }\end{array}$} & $\begin{array}{l}\text { Las formas organizativas en } \\
\text { las que participan desarrollan } \\
\text { procesos que promueven } \\
\text { espacios de aprendizaje y } \\
\text { transición agroecológica entre } \\
\text { productores }\end{array}$ \\
\hline $\begin{array}{l}\text { Otras formas de } \\
\text { evidenciamiento }\end{array}$ & \multicolumn{3}{|c|}{$\begin{array}{l}\text { Visitas a las unidades de producción (dias de campo o visitas } \\
\text { programadas); videos, fotos, relatos; talleres, y charlas que } \\
\text { abordan temas vinculados con cada proceso }\end{array}$} \\
\hline
\end{tabular}

Fuente: elaboración propia

El caso del Macuilli Teotzin se diferencia del resto por el tipo de relación que ha entablado con instituciones oficiales y por los efectos que esta puede tener en el largo plazo frente a la participación de nuevos productores y consumidores. Para algunos de sus participantes, este tipo de vínculos representa un logro y una oportunidad de acceder a otro tipo de mercados, mientras que para otros se trata de la implementación de mecanismos de control que no favorecen a pequeños y medianos productores, pues generan desconfianza frente a quienes no logran obtener la certificación. Además, se trata de PdC diseñados para certificar hortalizas, frutales, café y miel, por lo que se deja de lado la amplia oferta de productos transformados, que exige otro tipo de mecanismos de certificación, lo que da lugar a desigualdades entre los participantes.

Entre otros mecanismos de certificación diseñados por los mercados estudiados, sobresalen los sellos, las etiquetas y las categorías, a través de los cuales se resaltan las características de los productos y los productores, 
mecanismos que también sirven para motivar diálogos y relaciones entre los participantes en torno a las propiedades de los alimentos, recetas y usos. También, es usual que se realicen charlas, talleres, degustaciones y visitas a las unidades de producción que buscan dar a conocer los procesos de producción implementados por los participantes.

Dentro de estos mecanismos, identificamos que el principio de confianza es un pilar esencial de las relaciones sociales que allí se dan. En el caso del Alternativo de Puebla, se han diseñado PdC basados en otras formas de "evidenciamiento", como lo conceptualizan los propios actores:

Nuestro objetivo fue trabajar con un sistema de evidenciamiento que no iba a ser la certificación, sino que íbamos a buscar nuestras propias maneras de evidenciar que somos orgánicos, buscar una comunicación directa con los consumidores, dentro del mercado o fuera de él, haciendo visitas y conociendo al productor; lo orgánico está muy basado en la confianza y más en este tipo de mercados. (Productor del Alternativo de Puebla, comunicación personal, mayo de 2016)

En esta sintonía, los mercados ubicados en Colombia han recuperado como eje de su propuesta el precepto constitucional de la buena fe -manifestado en el artículo 83 constitucional-, el cual es integrado a partir de la reivindicación de la voz del campesinado: "Si el campesino me dice que produce limpio es porque está produciendo limpio; es decir, recuperar el valor de la palabra, y en Colombia es un precepto constitucional el de la buena fe” (líder campesino de Mercados Campesinos, comunicación personal, mayo de 2016).

A la confianza y la credibilidad sobre las que se basan y, al mismo tiempo, que estimulan estos PdC, se agregan elementos que politizan las prácticas, los discursos y las relaciones entre los participantes de los mercados. Por lo tanto, lo expresado por un líder y productor campesino en Colombia cobra importancia frente al objetivo que debe tener este tipo de mercados, que es, más allá de la posibilidad de certificar o no los productos, la habilitación y la reivindicación del campesinado en contextos donde sus prácticas y saberes han sido negados e invisibilizados: "Lo que buscamos es rescatar esos saberes, pero con un análisis político muy profundo, que cuestiona la dependencia que se va generando de paquetes [tecnológicos], semillas, mercados donde los que perdemos somos los campesinos” (líder y productor de Mercados Campesinos, comunicación personal, junio de 2016).

Ya sea a través de la implementación de PdC diseñados a partir del marco legal vigente, de estrategias propias de cada mercado o bien a partir de la reivindicación de relaciones basadas en la confianza, el objetivo de estos espacios gira en torno a la transformación y recuperación de los procesos productivos. Por lo tanto, con sus retos y contradicciones, estos mecanismos estimulan procesos acordes a los contextos en los que se ubican y a las necesidades de sus participantes, lo que a su vez les permite tomar distancia de recetas y normas preestablecidas por "expertos" que desconocen las realidades de sus contextos.

Se desarrollaron procesos para que cumplieran unos requisitos mínimos para estar dentro del proceso; paso a paso se fue logrando una cierta cualificación, pero también había una discusión, no podemos convertir al campesino o a la campesina: si ellos vienen con sus uñas llenas de tierra es porque trabajan la tierra, no podemos exigirles que estén impecables y que empiecen a producir de cierta manera, porque o si no los estaríamos obligando a ser lo que no son. (Líder de Mercados Campesinos, comunicación personal, junio de 2017)

Las estrategias de estas experiencias han apuntado a la transmisión de conocimientos, el diálogo de saberes entre productores y las escuelas campesinas, generando procesos de transición más eficientes que la implementación de manuales y reglamentos que no incorporan los contextos en los que se ubica el campesinado. En este sentido, los mercados analizados coinciden en su crítica al sistema agroalimentario dominante y plantean transformaciones desde espacios locales que reafirman su carácter alternativo. Asimismo, estimulan y afianzan las relaciones sociales, la relación campo-ciudad, la soberanía alimentaria y la recuperación de prácticas de cuidado de semillas e historias que permiten la reivindicación del campo y del campesinado.

La gente aprendió a valorar, a respetar el espacio de Mercados Campesinos, darse cuenta que son una fuente de identidad, de intercambio cultural, de valorar y sensibilizar, como un espacio muy enriquecedor para todos, tanto para la ciudad como para los productores y los consumidores. (Lideresa de Mercados Campesinos, comunicación personal, mayo de 2016) 
De esta manera, los efectos de los PdC en los mercados locales alternativos no solo rebasan la generación de condiciones favorables y diferenciadas para el intercambio de la producción, sino que permiten articular las etapas del proceso productivo y conectar a sus distintos actores, con lo que contribuyen a la configuración de redes y espacios de diálogo, dentro y fuera del mercado.

\section{Discusión}

En sus semejanzas y diferencias, las experiencias estudiadas muestran cómo se disputan los sentidos de las etapas del proceso productivo frente al mercado, al cual "se lo resignifica como espacio social, de encuentro y de lucha, confrontando la lógica hegemónica del mercado tradicional” (Caballero et al., 20ro, p. 39) a partir del diseño de estrategias innovadoras para resolver problemáticas y necesidades. Al mismo tiempo, en estos espacios se logran fortalecer procesos organizativos que rechazan las estructuras homogeneizantes impuestas por los sistemas alimentarios (Rossi y Brunori, 2010; Schwentesius, Gómez y Nelson, 2013), reflejadas, entre otras cosas, en la concentración del consumo en solo ig cultivos y 8 especies ganaderas (Montagut y Dogliotti, 2006).

La politización de las prácticas de producción, consumo y comercialización instituye intercambios basados en lazos sociales de confianza, cercanía, solidaridad y cooperación, lo que dinamiza estrategias alternativas apropiadas a cada contexto mediante diálogos que fortalecen cada experiencia (Sevilla y Soler, 20I2).

Los intercambios cara a cara fungen como una de las principales características de los mercados alternativos, pues, ante la ausencia de sellos y etiquetas oficiales, el diálogo y la confianza son los principales referentes para el consumidor (Santana, 2008). En ese sentido, los consumidores y productores otorgan a su participación en los mercados valores adicionales al económico; los $\mathrm{PdC}$ son una clara expresión de lo anterior, en la medida en que al alejarse -en diferentes intensidades- de los mecanismos oficiales, logran una reconfiguración de los términos frente a los debates en torno a los permisos, los sellos y las etiquetas como estrategias de control y vigilancia externa.

Por lo tanto, los PdC implementados por estos mercados constituyen alternativas al sistema agroalimentario, en la medida en que logran innovar en la construcción de relaciones de confianza como un elemento central de los procesos de intercambio. Al diseñar formas innovadoras de certificación basadas en estrategias de evidenciamiento, buena fe, confianza y respeto - que a su vez se construyen a partir de las características particulares de cada mercado y su contexto-, posibilitan la participación de productores excluidos del mercado convencional.

Sin embargo, esta diferenciación no alcanza, del todo, a favorecer de manera contundente y ampliada a los pequeños y medianos productores, pues este tipo de estrategias son cooptadas por otros espacios de producción y circulación de productos alternativos que reproducen relaciones sociales mediadas exclusivamente por los intercambios económicos.

En contraste con lo anterior, las iniciativas estudiadas expresan sentidos muy potentes en los que lo alternativo se expresa en la relación que tienen productoras y productores con la tierra, en la transmisión y reivindicación de conocimientos tradicionales, en los espacios por donde circulan los alimentos, en la diversidad de alimentos que se cultivan y se consumen - esto tiene que ver con la autonomía y la soberanía de cada productor sobre su unidad de producción-, en el rescate de productos y recetas y en el tipo de información y saberes que circulan entre productores y consumidores, entre otras. 


\section{Conclusiones}

En este artículo se identificaron y analizaron los mecanismos diseñados por cuatro mercados locales alternativos que identificaron el acceso a mercados favorables entre sus principales retos y que, por razones diversas, han implementado procesos de certificación de sus prácticas productivas. Su implementación revela formas heterogéneas de dinamizar estrategias similares que responden a las características de sus contextos y al tipo de participantes que las integran.

$\mathrm{Al}$ mismo tiempo, estos $\mathrm{PdC}$ ponen en juego procesos complementarios que no se agotan en la calidad de los productos ofertados, sino que visibilizan formas de articular las prácticas y los sujetos que las dinamizan a partir de resistencias y reivindicaciones frente a problemáticas mucho más amplias, representadas en el sistema agroalimentario dominante.

El cuestionamiento que hacen estos espacios al sistema agroalimentario va más allá de lo material-económico y se manifiesta en la consolidación de procesos de autonomía individual y colectiva construidos a partir del reconocimiento y la reafirmación de sus participantes en torno a preocupaciones comunes que convergen en la alimentación y los procesos productivos. En ese sentido, cada experiencia puede ser interpretada como una conquista espacial y simbólica en la disputa por otro tipo de relaciones sociales, económicas y políticas, principalmente en contextos locales y regionales en los que se ubican pero que tienen expresiones más amplias a nivel nacional e internacional.

En un contexto más amplio, estos mercados abonan a la reivindicación de pequeños y medianos productores, reconstruyendo la ficción de las etiquetas como garantía de calidad y revalorando la palabra como mecanismo principal de los intercambios. Con ello, visibilizan y cuestionan una lógica en la que, mientras este tipo de productores deben demostrar su inocencia para poder ingresar al mercado, las grandes cadenas de producción y distribución de alimentos saturan los estantes de los supermercados de alimentos con etiquetas que hacen grandes recorridos o con productos sin etiquetas que atentan contra la salud y el ecosistema.

Reconocer el origen, la heterogeneidad y las tensiones de las experiencias que se vienen consolidando ayuda a no idealizarlas, tanto entre quienes las integran como entre los que indagamos sus propuestas con el objetivo de contribuir a su fortalecimiento.

Finalmente, pese a sus contradicciones y conflictos, tanto a nivel local como regional, estos espacios están buscando articular diversas reivindicaciones de pequeños y medianos productores que no solo plantean otras formas de relacionarse con los mercados, sino que, en su confluencia, pujan por otros mercados posibles.

\section{Referencias}

Barriga, L. (2016). Redes alimentarias alternativas, contribuciones a la reconexión del consumidor con la alimentación. Desarrollo Rural. IPDRS, (30). Recuperado de https://www.ocaru.org.ec/index.php/debate-rural/agroindustria-y-extractivas/item/7382-redes-alimenta rias-alternativas-contribuciones-a-la-reconexion-del-consumidor-con-la-alimentacion

Beckie, M., Kennedy, E., y Wittman, H. (20I2). Scaling up alternative food networks: Farmers' markets and the role of clustering in western Canada. Agriculture and Human Values, 29(3), 333-345. https://www.doi.org/ 10.1007/S10460-0I2-9359-9

Caballero, L., Dumraf, S., Gonzales, E., Mainella, F., y Moricz, M. (2010). Los procesos organizativos de la agricultura y la creación de ferias y mercado de economía social. Otra Economía, 4(7), 43-66. Recuperado de https://www.revistas.unisinos.br/index.php/otraeconomia/article/view/1300/362 
Calle, Á., Soler, M., y Rivera, M. (20II). Soberanía alimentaria y agroecología emergente: la democracia alimentaria. En Á. Calle, Aproximaciones a la democracia radical (pp. 213-238). Barcelona: Icaria.

Calle, Á., Vara, I., y Cuéllar, M. (20I3). La transición social agroecológica. En M. Cuéllar, Á. Calle y D. Gallar (eds.), Procesos hacia la soberanía alimentaria. Perspectivas y prácticas desde la agroecología política (pp. 8I-I02). Barcelona: Icaria.

Carlisle, L. (2015). Audits and agrarianism: The moral economy of an alternative food network. Elementa: Science of the Anthropocene, 3(2I). https://www.doi.org/I0.I2952/journal.elementa.000066

Coraggio, J. (2004). La gente o el capital. Desarrollo local y economía del trabajo. Buenos Aires: Espacio.

Crespo, B., y Sabín, F. (2014). Los mercados sociales. La economía solidaria en acción transformadora. Documentación Social (174), 95-II6. Recuperado de https://www.socioeco.org/bdf_fiche-document-4037_es. html

Cuéllar, M. (2010). La certificación ecológica como instrumento de revalorización de lo local: los Sistemas Participativos de Garantía en Andalucía. En R. Fernández-Baca Casares (dir.), M. Soler Montiel y C. Guerrero Quintero (coords.), Patrimonio cultural en la nueva ruralidad andaluza (pp. 284-925). Sevilla: Junta de Andalucía, Consejería de Cultura, Instituto Andaluz del Patrimonio Histórico.

Delgado, M. (2010). El sistema agroalimentario globalizado: imperios alimentarios y degradación social ecológica. Revista de Economía Critica, (IO), 32-6I. Recuperado de https://www.researchgate.net/publication/227488I 63_El_sistema_agroalimentario_globalizado_imperios_alimentarios_y_degradacion_social_y_ecologica

Escobar, A. (2010). Territorios de diferencia: lugar, movimientos, vida, redes. Popayán: Envión.

Gil, J. (20I4). “Los límites del mercado: reflexiones sobre economía, antropología y democracia”, de Karl Polanyi. Encrucijadas: Revista Crítica de Ciencias Sociales, 7, I9I-I94. Recuperado de https://www.encrucijadas.org /index.php/ojs/article/view/36

Giraldo, O., y Rosset, P. (2016). La agroecología en una encrucijada: entre la institucionalidad y los movimientos sociales. Guaju, 2(I), I4-37. https://www.dx.doi.org/10.5380/guaju.v2iı.4852I

Gómez, M., Schwentesius, R., Ortigoza, J., y Gómez, L. (2010). Situación y desafíos del sector orgánico de México. Revista Mexicana de Ciencias Agrícolas, I(4), 593-608. Recuperado de https://www.scielo.org.mx/scielo.ph p?script=sci_arttext\&pid=S2007-093420100004000II

Gracia, M. A. (2015). Indagar el campo de posibilidades de las experiencias de trabajo asociativo autogestionado. En M. A. Gracia, Trabajo, reciprocidad y re-producción de la vida. Experiencias colectivas de autogestión y economía solidaria en América Latina (pp. 17-56). Buenos Aires: Miño y Dávila.

Kay, S. (2016). Vinculación de los productores a pequeña escala con los mercados. Francia: Mecanismo de la Sociedad Civil (MSC). Recuperado de https://www.fao.org/fileadmin/templates/cfs/Docsi516/cfs43/CS M_Connecting_Smallholder_to_Markets_SP.pdf

Lahera, A. (1999). La crítica de la economía de mercado en Karl Polanyi: el análisis institucional como pensamiento para la acción. Reis, (86), 27-54. Recuperado de https://www.dialnet.unirioja.es/servlet/articulo?codigo=759 784

Lamine, C., Darolt, M., y Brandenburg, A. (2012). The civic and social dimensions of food production and distribution in alternative food networks in France and southern Brazil. International Journal of Agriculture and Food, 19(3), 383-40I.

León, G., Valdez, H., y Vásquez, V. (2003). Mercado Kantuta: ¿un mercado campesino? La Paz: Programa de Investigación Estratégica en Bolivia (PIEB). 
Long, N. (2007). Sociología del desarrollo: una perspectiva centrada en el actor. San Luis Potosí: El Colegio de San Luis, Centro de Investigaciones y Estudios Superiores en Antropología Social (Ciesas).

Maela. (20I2). Boletín mensual. Hoja a Hoja, (I4).

Malassis, L. (1973). Economie agro-alimentaire: economie de la consommation et de la production agroalimentaire. París: Cujas.

Montagut, X., y Dogliotti, F. (2006). Alimentos globalizados: soberanía alimentaria y comercio justo (vol. 235). Barcelona: Icaria.

Parrado, A., y Molina, J. (20I4). Mercados campesinos: modelo de acceso a mercados y seguridad alimentaria en la región central de Colombia. Bogotá: Oxfam.

Roldán-Rueda, H., Gracia, M. A., Santana, M., y Horbath, J. (2016). Los mercados orgánicos en México como escenarios de construcción social de alternativas. Polis, Revista Latinoamericana, 15(43), 581-605. https://w ww.dx.doi.org/10.4067/S0718-65682016000100027

Roldán-Rueda, H., y Gracia, M. A. (2015). Espacios de intercambio local de productos orgánicos en México. Panorama, tensiones y procesos de aprendizaje. En M. A. Gracia, Trabajo, reciprocidad y re-producción de la vida. Experiencias colectivas de autogestión y economía solidaria en América Latina (pp. 285-314). Buenos Aires: Miño y Dávila.

Rosset, P. (2007). Mirando hacia el futuro: la reforma agraria y la soberanía alimentaria. Revista Internacional de Ciencias Sociales, (26), I67-182. Recuperado de https://www.revistas.um.es/areas/article/view/II857I

Rossi, A., y Brunori, G. (2010). Drivers of transformation in the agro-food system. GAS as co-production of Alternative Food Networks. 9th European IFSA Symposium, Viena. Recuperado de https://www.ifsa.bok u.ac.at/cms/fileadmin/Proceeding2010/2010_WS4.4_Rossi.pdf

Santana, M. (2008). Reinventando el dinero. Experiencias con monedas comunitarias (Tesis de doctorado). Centro de Investigaciones y Estudios Superiores en Antropología Social (Ciesas), México.

Schwentesius, R., Gómez, M., y Nelson, E. (2013). La Red Mexicana de Tianguis y Mercados Orgánicos. Renovando sistemas de abasto de bienes de primera necesidad para pequeños productores y muchos consumidores. En Sistemas participativos de garantía. Estudios de caso en América Latina (pp. 2I-34). Alemania: Ifoam. Recuperado de https://www.ifoam.bio/sites/default/files/page/files/la_case_studies_co lor_print_fc_o.pdf

Sevilla, E., y Soler, M. (20I0). Agroecología y soberanía alimentaria: alternativas a la globalización agroalimentaria. En R. Fernández-Baca Casares (dir.), M. Soler Montiel y C. Guerrero Quintero (coords.), Patrimonio cultural en la nueva ruralidad andaluza (pp. 190-217). Sevilla: Junta de Andalucía, Consejería de Cultura, Instituto Andaluz del Patrimonio Histórico. Recuperado de https://www.institucional.us.es/compromiso/libreconf /docs/agroecologia.pdf

Soler, M., y Calle, A. (2010). Rearticulando desde la alimentación: canales cortos de comercialización en Andalucía. En R. Fernández-Baca Casares (dir.), M. Soler Montiel y C. Guerrero Quintero (coords.), Patrimonio cultural en la nueva ruralidad andaluza (pp. 258-283). Sevilla: Junta de Andalucía, Consejería de Cultura, Instituto Andaluz del Patrimonio Histórico.

Tarditti, M. (2012). Redes alimentarias alternativas y soberanía alimentaria. Posibilidades para la transformación del sistema agroalimentario dominante (Tesis de Doctorado en Sociología). Universidad Autónoma de Barcelona, Barcelona. Recuperado de https://www.socioeco.org/bdf_fiche-document-3623_es.html

Toledo, V. (1992). Toda la utopía: el nuevo movimiento ecológico de los indígenas y campesinos de México. En J. Moguel, C. Botey y L. Hernández (coords.), Autonomía y nuevos sujetos sociales en el desarrollo rural. Ciudad de México: Siglo XXI. 
Yin, R. (1994). Investigación sobre estudio de casos. Diseño y métodos. Applied Social Research Methods Series, 5(2). Recuperado de https://www.panel.inkuba.com/sites/2/archivos/YIN\%20ROBERT\%20.pdf

Zamilpa, J. (20I4). Fortalecimiento del sector orgánico de México: aprendiendo de la experiencia de la Unión Europea. Michoacán: Universidad Michoacana de San Nicolás de Hidalgo, Instituto de Investigaciones Económicas y Empresariales.

Zemelman, H. (2006). El conocimiento como desafío posible. Ciudad de México: Instituto Politécnico Nacional.

\section{Notas}

* Artículo de investigación

Licencia Creative Commons CC BY-4.o 